\title{
Theory of Nonlinear Viscoelasticity in Temporarily Crosslinked Polymer Systems
}

\author{
Shizuo HAYASHI \\ Department of Physics, Faculty of General Studies, Gunma University, \\ Aramaki 4-2, Maebashi, Japan
}

(Received August 13, 1984)

\begin{abstract}
A nonlinear chain breakage-and-reformation network theory is presented using a temporarily crosslinked network model. The main assumptions are as follows: (1) The number of segments in a polymer chain is constant; (2) a renewed segment is reformed in the stress free state; (3) the length of a segment is constant and (4) segments are Gaussian chains. Third assumption gives rise to the nonlinear theory. The constitutive equation is obtained as a natural extension of linear equation. The following predictions are obtained. The relaxation modulus is the product of the time dependent and strain dependent terms for both shear and elongation. At the onset of flow, the shear stress growth shows overshoot at high shear rate, but not the normal stress growth, the elongational stress grows monotonously and the elongational viscosity is nearly proportional to the inverse of the elongation rate.

KEY WORDS Nonlinear Viscoelasticity / Network Theory / Chain Breakage-Reformation / Stress Relaxation / Stress Growth /
\end{abstract}

The viscoelastic behavior of polymer in the melt and concentrated solutions is generally nonlinear and has been investigated in terms of nonlinear theory as it has progressed recently.

One theoretical approach is the strudy of phenomenological constitutive equations, ${ }^{1-23}$ which generally have integral formulas. Many authors have presented various constitutive equations $s^{1-13}$ and investigated their applicability. ${ }^{15-19}$ The strain dependent constitutive equation containing the invariant of strain tensor in its kernel seems to have the broadest application. $^{15-19}$

Other theoretical approaches are based on molecular theories. One such theory is the network theory based on a temporarily crosslinked network model, ${ }^{28-38}$ and another is the cage field theory developed by Doi and Edwards, ${ }^{25-27}$ based on a model in which a polymer chain moves independently in a cage, the three dimensional mean field pattern being imposed by other chains.

The network theory can be classified into two types. One is the chain breakage-andreformation theory in which the relaxation processes originate in chain breakage and chain reformation, ${ }^{28-30}$ and the other is the chain slip theory. ${ }^{31-38}$ Both of these have the same formalism in the linear theory. ${ }^{31}$

Yamamoto's chain breakage-and-reformation theory has a quite general formalism and is substantially nonlinear theory. Takano applied Yamamoto's theory to certain practical problems and successfully explained some nonlinear viscoelastic properties of concentrated polymer systems. ${ }^{34,35}$

The chain slip theory is extended to a nonlinear theory, in which the rates of chain breakage and reformation in a deformed state differ from those in a stress free state, ${ }^{36-38}$ that is, it is a combined theory of the chain slip theory and chain breakage-and-reformation theory. The theory explains fairly well some nonlinear viscoelastic phenomena, for instance, non-Newtonian viscosity, stress overshoot phenomena at the onset of steady shear 
flow, but not the stress relaxation phenomena at large deformation for periods of long times.

The cage field theory was developed using the primitive chain concept, the tube model, in which a polymer chain moves in a confined tube-like region surrounding it. ${ }^{25-27}$ Doi and Edwards applied their theory to some typical deformations and successfully explained nonlinear viscoelastic properties. The cage field theory leads to the chain slip theory based on a network model having temporary junctions if the tube model is replaced by a group of small rings through which the chain can slip freely.

In this report, we present a chain breakageand-reformation network theory.

We consider a temporarily crosslinked network model in which a polymer chain moves through junctions, and when the terminal part of the chain slips out through the junction points, segments are lost from the network and a long dangling segment is formed. A segment is a part of the polymer chain constituting the network structure. When the long dangling segment is recrosslinked to other chains, new segments are formed. This process corresponds to tube disappearance and renewal in the tube.

In developing the theory, we make following assumptions. (1) The number of segments in a polymer chain is kept constant during deformation. (2) A segment is reformed in the stress free state, the so called natural state. (3) The transformation of the end-to-end vector of a segment by deformation is pseudo-affine; that is, the orientation of a segment is equal to that in the case of affine transformation but the segment length is constant. (4) Segment are Gaussian chains.

Assumption (3) gives rise to the nonlinear theory and the other assumptions are usually applicable to the network theory.

The theory may be applied to typical deformation, stress rerelaxation at a large constant deformation, stress growth at the onset of a stationary flow and shear and elongation flows.

\section{CONSTITUTIVE EQUATION}

Let the segment distribution function be $f(\boldsymbol{r}, t)$ the number of segments having the endto-end vector $\boldsymbol{r}$ at time $t$ in a unit volume of the network structure, and the segment distribution function at the stress free state be $f_{0}(\boldsymbol{r}, \mathbf{t})$. The relation between the number of segments in unit volume and the distribution function is given as

$$
\int f(\boldsymbol{r}, t) \mathrm{d} \boldsymbol{r}=\int f_{0}(\boldsymbol{r}, t) \mathrm{d} \boldsymbol{r}=v
$$

where the integral is the volume integral, and $v$ is kept constant during deformation by assumption 1 . The time revolution of the segment distribution function satisfies the diffusion equation equivalent to Yamamoto's equation, ${ }^{8}$

$$
\frac{\partial f(\boldsymbol{r}, t)}{\partial t}=-\nabla(\dot{\boldsymbol{r}} f(\boldsymbol{r}, t))-K f(\boldsymbol{r}, t)+g_{0}(\boldsymbol{r}, t)
$$

where $\dot{\boldsymbol{r}}=\mathrm{d} \boldsymbol{r} / d t, K$ is the chain breakage constant and $g_{0}(\boldsymbol{r}, \mathbf{t})$ is the segment generating function. Since segments are created in the stress free state $g_{0}(\boldsymbol{r}, t)$ satisfies the relation (3) by assumption (1),

$$
\left.\int\left\{\int_{-\infty}^{t} g_{0}\left(\boldsymbol{r}, t^{\prime}\right) \mathrm{d} t^{\prime}\right)\right\} \mathrm{d} \boldsymbol{r}=K \int f_{0}(\boldsymbol{r}, t) \mathrm{d} \boldsymbol{r}
$$

Let the deformation tensor of the network structure at a time $t$ be $\gamma(t)$, the change of $r(t)$ after a time interval $\Delta t$ is satisfied by the relation

$$
r(t+\Delta t)=\frac{\gamma(t+\Delta t, t) \cdot r(t)}{|\gamma(t+\Delta t, t) \cdot u(t)|}
$$

in which

$$
\left.\begin{array}{l}
\boldsymbol{u}(t)=\boldsymbol{r}(t) / a \\
\gamma\left(t, t^{\prime}\right)=\gamma(t) \cdot \gamma^{-1}\left(t^{\prime}\right)
\end{array}\right\}
$$

where $a$ is the end-to-end distance of a segment, $\boldsymbol{u}$, the unit vector in the direction of $\boldsymbol{r}$ by the assumption (3), and $\gamma\left(t, t^{\prime}\right)$ the relative deformation tensor at a time $t$ with respect to a previous time $t^{\prime}$. Using eq $4, \dot{r}$ in eq 2 is 
obtained as

$$
\left.\begin{array}{l}
\dot{\boldsymbol{r}}=\dot{\gamma} \boldsymbol{r}-\frac{1}{2} \operatorname{Tr} .\left\{\left(\dot{\gamma}+\dot{\gamma}^{\dagger}\right) \cdot(\boldsymbol{u u})\right\} \cdot \boldsymbol{r} \\
\dot{\gamma}=(\mathrm{d} \gamma / \mathrm{d} t) \cdot \gamma^{-1}
\end{array}\right\}
$$

where $(\boldsymbol{u} \boldsymbol{u})$ is a dyad and $\dot{\gamma}$ is the deformation rate.

Since the segments are Gaussian, we obtain the stress $\sigma(t)$ as

$$
\boldsymbol{\sigma}(t)=\frac{3 k T}{a^{2}} \int(\boldsymbol{r} \boldsymbol{r}) f(\boldsymbol{r}, t) \mathrm{d} \boldsymbol{r}-P \mathbf{1}
$$

where $k$ is the Boltzmann constant, $T$, the absolute temperature, $P$, the internal pressure, and 1 the unit tensor.

Introducting the average of $(\boldsymbol{r} r)$ defined by

$$
\begin{aligned}
\langle(\boldsymbol{r} r)\rangle & =\frac{1}{v} \int(\boldsymbol{r} r) f(\boldsymbol{r}, t) \mathrm{d} \boldsymbol{r} \\
\langle(\boldsymbol{r} r)\rangle_{0} & =\frac{1}{v} \int(\boldsymbol{r} \boldsymbol{r}) f_{0}(\boldsymbol{r}, t) \mathrm{d} \boldsymbol{r}
\end{aligned}
$$

and the strain tensor $\alpha(t)$ defined by

$$
\langle(\boldsymbol{r} r)\rangle=\frac{a^{2}}{3} \alpha(t)
$$

the stress is given by

$$
\boldsymbol{\sigma}(t)=v k T \alpha(t)-P \mathbf{1}
$$

In order to obtain a constitutive equation, we must find a equation satisfied by $\alpha(t)$.

The equation satisfying the time evolution of $\alpha(t)$ is obtained using the diffusion equation and eq 12 ,

$$
\dot{\boldsymbol{\alpha}}=\frac{3}{v a^{2}} \int(\boldsymbol{r} \boldsymbol{r}) \frac{\partial f(\boldsymbol{r}, t)}{\partial t} \mathrm{~d} \boldsymbol{r}
$$

and $\alpha(t)$ is satisfied by eq 13

$\dot{\gamma} \cdot \boldsymbol{\alpha}+\boldsymbol{\alpha} \cdot \dot{\gamma}^{+}-\dot{\alpha}-\operatorname{Tr} .\left\{\left(\dot{\gamma}+\dot{\gamma}^{\dagger}\right) \cdot \boldsymbol{\alpha} / 3\right\} \cdot \boldsymbol{\alpha}=\frac{1}{\tau}(\boldsymbol{\alpha}-\mathbf{1})$

where $\tau=1 / K$ is the relaxation time for chain breakage and the approximation used for the average is

$$
\begin{aligned}
& \left\langle\operatorname{Tr} .\left\{\left(\dot{\gamma}+\dot{\gamma}^{\dagger}\right) \cdot(\boldsymbol{u u})\right\} \cdot(\boldsymbol{u u})\right\rangle \\
& \quad=\operatorname{Tr} .\left\{\left(\dot{\gamma}+\dot{\gamma}^{\dagger}\right)\langle(\boldsymbol{u} u)\rangle\right\} \cdot\langle(\boldsymbol{u} u)\rangle
\end{aligned}
$$

From eq 13 we obtain

$$
\left.\begin{array}{rl}
\alpha(t) & =\int_{-\infty}^{t} \frac{\mathrm{e}^{-\left(t-t^{\prime}\right) / \tau}}{\tau} \frac{3 \lambda\left(t, t^{\prime}\right)}{\operatorname{Tr} . \lambda\left(t, t^{\prime}\right)} \mathrm{d} t^{\prime} \\
\lambda\left(t, t^{\prime}\right) & =\gamma\left(t, t^{\prime}\right) \cdot \gamma^{\dagger}\left(t, t^{\prime}\right)
\end{array}\right\}
$$

The method of obtaining eq 15 is given in the appendix $\mathrm{I}$.

We obtain the constitutive equation from eq 11 and eq 15 ,

$$
\left.\begin{array}{l}
\boldsymbol{\sigma}(t)=G_{0} \int_{-\infty}^{t} \frac{\mathrm{e}^{-\left(t-t^{\prime}\right) / \tau}}{\tau} \frac{3 \lambda\left(t, t^{\prime}\right)}{\operatorname{Tr} . \lambda\left(t, t^{\prime}\right)} \mathrm{d} t^{\prime}-P \mathbf{1} \\
G_{0}=v k T
\end{array}\right\}
$$

Equation 16 is equivalent to Doi-Edwards' equation in the case of the longest relaxation time. The relation between them is shown in the appendix II.

\section{APPLICATION}

In the following, application of the constitutive equation to various typical deformations is considered.

Stress Relaxation at a Large Constant Deformation

\section{a. Shear Deformation}

When the constant shear deformation $\gamma_{0}$ is given for the system, at $t=0, \gamma(t)$ is expressed as

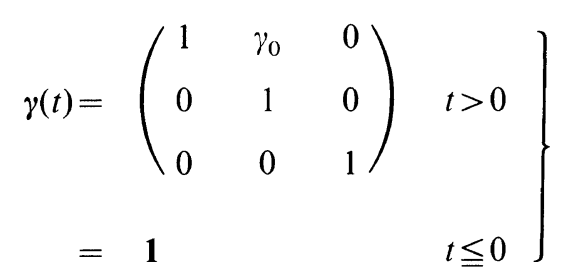

and $\lambda\left(t, t^{\prime}\right)$ as 


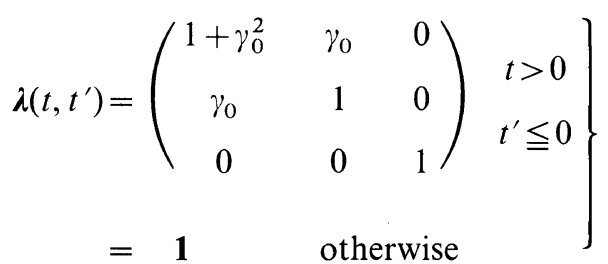

The shear stress $\sigma_{x y}$ and first normal stress difference $\Delta \sigma_{n}$ are given by

$$
\begin{gathered}
\sigma_{x y}(t)=G_{0} \gamma_{0} \mu(t) f_{\mathrm{sh}}\left(\gamma_{0}\right) \\
\Delta \sigma_{n}(t)=G_{0} \gamma_{0}^{2} \mu(t) f_{\mathrm{sh}}\left(\gamma_{0}\right) \\
\mu(t)=\mathrm{e}^{-t / \tau} \\
f_{\mathrm{sh}}\left(\gamma_{0}\right)=\left(1+\gamma_{0}^{2} / 3\right)^{-1}
\end{gathered}
$$

The shear relaxation modulus $G_{\mathrm{sh}}\left(\gamma_{0}, t\right)$ is given by

$$
G_{\mathrm{sh}}\left(\gamma_{0}, t\right)=G_{0} \mu(t) f_{\mathrm{sh}}\left(\gamma_{0}\right)
$$

\section{b. Elongational Deformation}

When the system is elongated in one direction by a factor $\xi_{0}$ and the volume is not changed by deformation, $\gamma(t)$ is expressed as

$$
\left.\begin{array}{rl}
\gamma(t) & =\left(\begin{array}{ccc}
\xi_{0} & 0 & 0 \\
0 & \xi_{0}^{-1 / 2} & 0 \\
0 & 0 & \xi_{0}^{-1 / 2}
\end{array}\right) t>0 \\
& =1
\end{array}\right\}
$$

and $\lambda\left(t, t^{\prime}\right)$ as

$$
\begin{aligned}
& \left.\lambda\left(t, t^{\prime}\right)=\left(\begin{array}{ccc}
\xi_{0}^{2} & 0 & 0 \\
0 & \xi_{0}^{-1} & 0 \\
0 & 0 & \xi_{0}^{-1}
\end{array}\right) \begin{array}{c}
t>0 \\
t^{\prime} \leqq 0
\end{array}\right\} \\
& =1 \text { otherwise }
\end{aligned}
$$

and the tensile modulus $G_{\mathrm{el}}\left(\xi_{0}, t\right)$ is given as

$$
G_{\mathrm{el}}\left(\xi_{0}, t\right)=G_{0} \mu(t) f_{\mathrm{el}}\left(\xi_{0}\right)
$$

Equations 23 and 29 show that the relaxation modulus is expressed as the product of the time dependent and strain dependent terms. A logarithmic plot of modulus against time is shown in Figure 1, in which the modulus is expressed by a master curve when the plotted curves are shifted along the modulus axis, and the shift factors are $\log f_{\mathrm{sh}}\left(\gamma_{0}\right)$ for the shear and $\log f_{\mathrm{el}}\left(\xi_{0}\right)$ for the elongation, respectively.

Stress Growth at the Onset of a Steady Flow a. Shear Flow

When a shear flow with a constant shear rate $\kappa$ starts at $t=0, \gamma(t)$ is expressed as

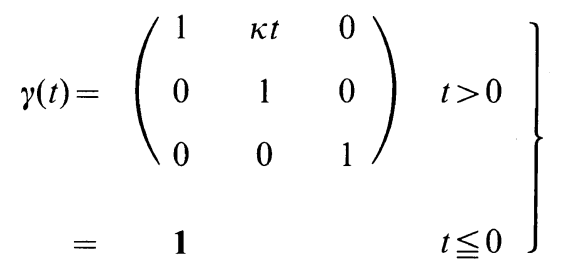

and $\lambda\left(t, t^{\prime}\right)$ as

$$
\begin{aligned}
\lambda\left(t, t^{\prime}\right) & =\left(\begin{array}{ccc}
1+\kappa^{2}\left(t-t^{\prime}\right)^{2} & \kappa\left(t-t^{\prime}\right) & 0 \\
\kappa\left(t-t^{\prime}\right) & 1 & 0 \\
0 & 0 & 1
\end{array}\right) t, t^{\prime}>0 \\
& =\left(\begin{array}{ccc}
1+\kappa^{2} t^{2} & \kappa t & 0 \\
\kappa t & 1 & 0 \\
0 & 0 & 1
\end{array}\right) \begin{array}{c}
t>0 \\
t^{\prime} \leqq 0
\end{array} \\
& =1
\end{aligned}
$$

The shear stress $\sigma_{x y}(\kappa, t)$ and first normal stress difference $\Delta \sigma_{\mathrm{n}}(\kappa, t)$ are given by

$$
\begin{gathered}
\sigma_{x y}(\kappa, t)=G_{0} F_{\mathrm{s}}(\kappa, t) \\
F_{\mathrm{s}}(\kappa, t)=\int_{0}^{\kappa t} \mathrm{e}^{-x / \kappa \tau} \frac{\mathrm{d}}{\mathrm{d} x}\left(\frac{x}{1+x^{2} / 3}\right) \mathrm{d} x
\end{gathered}
$$




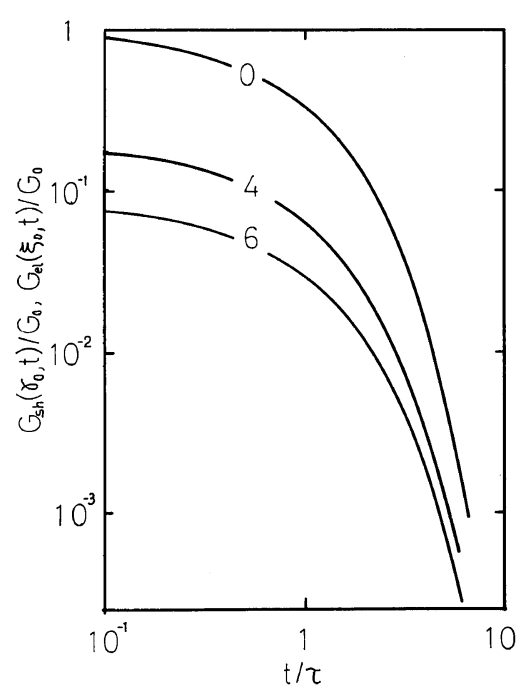

Figure 1. Relaxation modulus versus time relation. The relaxation moduli for different deformations are plotted against time. The number on each curve indicates the value of $\gamma_{0}$ for shear and $\xi_{0}$ for elongation. Though $f_{\mathrm{sh}}\left(\gamma_{0}\right)$ and $f_{\mathrm{el}}\left(\xi_{0}\right)$ are different for the same values of $\gamma_{0}$ and $\xi_{0}$, the difference is small and does not appear in the drawn curves. The curves can be superposed on a master curve when they are shifted along the moulus-axis.

$$
\begin{gathered}
\Delta \sigma_{\mathrm{n}}(\kappa, t)=G_{0} F_{\mathrm{n}}(\kappa, t) \\
F_{n}(\kappa, t)=\int_{0}^{\kappa t} \mathrm{e}^{-x / \kappa \tau} \frac{\mathrm{d}}{\mathrm{d} x}\left(\frac{x^{2}}{1+x^{2} / 3}\right) \mathrm{d} x
\end{gathered}
$$

In eq 33 , the following relations

$$
\left.\begin{array}{rlrl}
\frac{\mathrm{d}}{\mathrm{d} x}\left(\frac{x}{1+x^{2} / 3}\right) & >0 & \text { for } & 0<x<\sqrt{3} \\
=0 & x=\sqrt{3} \\
<0 & x>\sqrt{3}
\end{array}\right\}
$$

are satisfied. The integrand in eq 33 is positive for $0<x<3$ and accordingly, $F_{\mathrm{s}}(\kappa, t)$ increases with time up to $\kappa t=\sqrt{3}$. The integrand is negative for $x>3$ and $F_{\mathrm{s}}(\kappa, t)$ decreases when $\kappa t>\sqrt{3}$. Therefore, $F_{\mathrm{s}}(\kappa, t)$ has a maximum at $t_{\mathrm{M}}=\sqrt{3} / \kappa$; that is, the stress overshoot appears at $t=t_{\mathrm{M}}$. When the shear stress is plotted against the shear deformation $\gamma(t)=\kappa t$, the maximum stress always appears at the position $\gamma(t)=\sqrt{3}$, irrespective of the shear rate, and

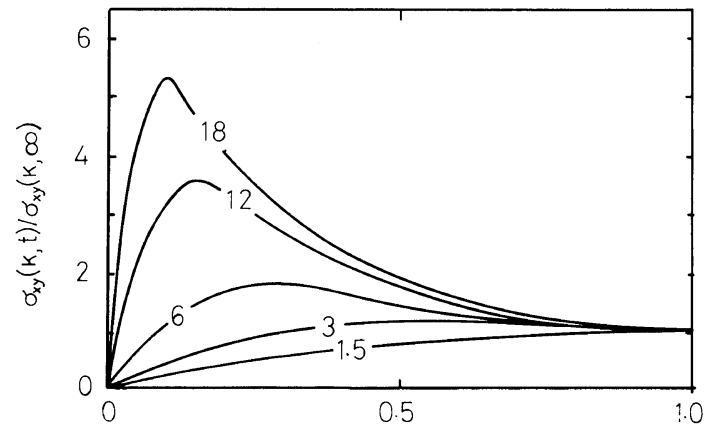

Figure 2. Shear stress growth at the onset of shear flow. When the stress growth is plotted against time, the time required to reach the maximum value of stress becomes shorter as the shear rate increases. The number on each curve indicates the value of $\kappa \tau$.

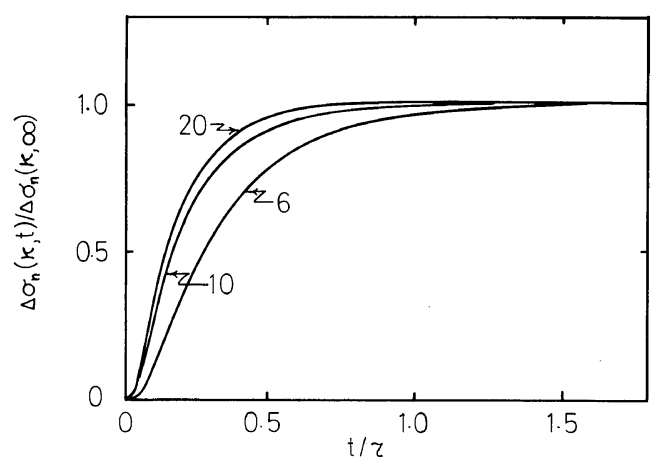

Figure 3. Normal stress growth at the onset of shear flow. Normal stress grows monotonously with time for each shear rate. The notations are the same as those in Figure 2.

accordingly, $t_{\mathrm{M}}$ decreases with increasing shear rate. If $t_{\mathrm{M}}$ is larger than $\tau$, the shear stress attains a constant steady value before reach a maximum, since the stress takes on a steady value almost at the end of the time $\tau$. Therefore, $\kappa \tau \simeq \sqrt{3}$ is the critical condition determining whether stress overshoot will appear.

The shear stress corresponding to $t=\infty$ gives the steady shear stress and the steady shear viscosity is given by

$$
\eta(\kappa)=\frac{G_{0} F_{\mathrm{s}}(\kappa, \infty)}{\kappa} \simeq \frac{G_{0} \tau}{\left(1+\kappa^{2} \tau^{2} / 3\right)}
$$

$\eta(\kappa)$ is a decreasing function of the shear 
rate.

The integrand in eq 35 is always positive and vanishes at infinity, and accordingly, $\Delta \sigma_{n}(\kappa, t)$ increases monotonously with time and tends to take on a steady value for each shear rate.

The shear stress versus time relation is shown in Figure 2 and the normal stress versus time relation in Figure 3 .

\section{b. Elongational Flow}

When a steady simple elongational flow with a constant elongation rate $a$ is given at $t=0$, $\gamma(t)$ is expressed as

$$
\begin{aligned}
& \gamma(t)=\left(\begin{array}{ccc}
\mathrm{e}^{a t} & 0 & 0 \\
0 & \mathrm{e}^{-a t / 2} & 0 \\
0 & 0 & \mathrm{e}^{-a t / 2}
\end{array}\right) t>0 \\
& =1 \\
& t \leqq 0
\end{aligned}
$$

Where we assume that the volume does not change at the deformation, and $\lambda\left(t, t^{\prime}\right)$ becomes

$$
\begin{aligned}
\lambda\left(t, t^{\prime}\right) & =\left(\begin{array}{ccc}
\mathrm{e}^{2 a\left(t-t^{\prime}\right)} & 0 & 0 \\
0 & \mathrm{e}^{-a\left(t-t^{\prime}\right)} & 0 \\
0 & 0 & \mathrm{e}^{-a\left(t-t^{\prime}\right)}
\end{array}\right) t, t^{\prime}>0 \\
& =\left(\begin{array}{ccc}
\mathrm{e}^{2 a t} & 0 & 0 \\
0 & \mathrm{e}^{-a t} & 0 \\
0 & 0 & \mathrm{e}^{-a t}
\end{array}\right) \begin{array}{l}
t>0 \\
t^{\prime} \leqq 0
\end{array} \\
& =1 \text { otherwise }
\end{aligned}
$$

The stress acting in the elongational direction becomes

$$
\begin{gathered}
\sigma_{x x}(a, t)=3 G_{0} F_{\mathrm{el}}(a, t) \\
F_{\mathrm{el}}(a, t)=\int_{0}^{t} \mathrm{e}^{-x / \tau} \frac{\mathrm{d}}{\mathrm{d} x}\left(\frac{1-\mathrm{e}^{-3 a x}}{1+2 \mathrm{e}^{-3 a x}}\right) \mathrm{d} x
\end{gathered}
$$

and increases monotonously. The stress versus time relation is shown in Figure 4. At a high elongation rate the stress increases more rap-

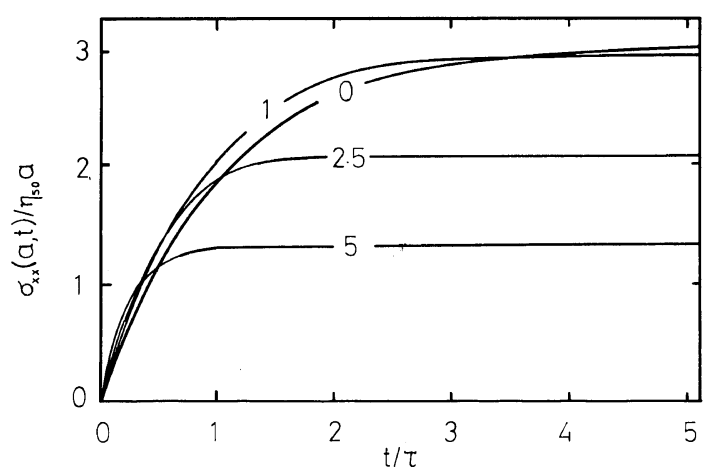

Figure 4. Stress growth at the onset of elongational flow. Stress grows monotonously with time for each elongation rate. Curves corresponding to different elongation rates intersect with each other. The number on each curve indicates the value of $a \tau$.

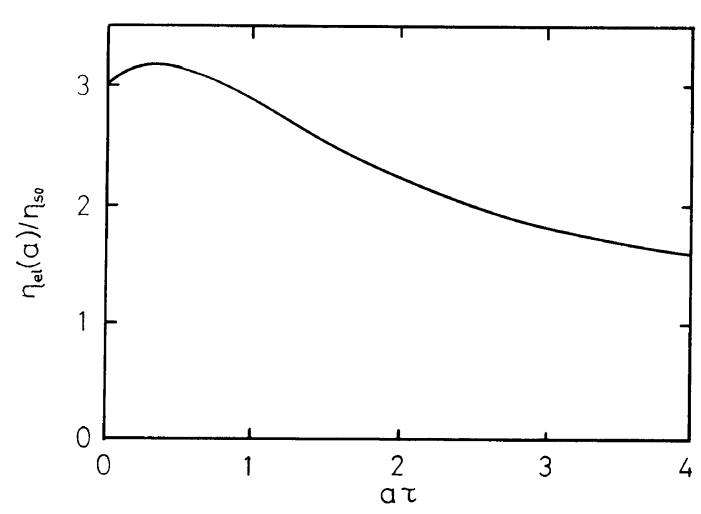

Figure 5. Elongational viscosity versus elongation rate relation. As the elongation rate increases, the elongational viscosity increases somewhat at the beginning and, after passing a maximum value, decreases nearly inversely proportional to the elongation rate.

idly at the beginning and tends to take on a lower steady value than when the elongation rate is low. That is, a curve for a high elongation rate crosses those for low elongation rates.

The elongational viscosity is defined by

$$
\eta_{\mathrm{el}}(a)=\frac{3 G_{0} F_{\mathrm{el}}(a, \infty)}{a}
$$

Since the zero shear viscosity $\eta_{\mathrm{so}}$ is equal to $G_{0} \tau$ by eq 37 , we have from eq 42 


$$
\left.\begin{array}{rl}
\frac{\eta_{\mathrm{el}}(a)}{\eta_{\mathrm{so}}} & \simeq \frac{3}{a \tau} \frac{1-\mathrm{e}^{-3 a \tau}}{1+2 \mathrm{e}^{-3 a \tau}} \\
& \simeq 3 \quad \text { for } \quad a \tau \ll 1 \\
& \propto a^{-1} \quad \text { for } \quad a \tau \gg 1
\end{array}\right\}
$$

and Trouton's relation is satisfied in the region of low elongation rate. When the elongation rate increases, the elongational viscosity $\eta_{\mathrm{el}}(a)$ increases a little in the beginning and passing a maximum it decreases nearly inversely proportional to the elongation speed in high elongation speed regions. The elongational viscosity versus elongation rate relation is shown in Figure 5.

\section{CONCLUDING REMARKS}

The present network theory of nonlinear viscoelasticity is classed as the chain breakageand-reformation theory in which reformed chains are recrosslinked in the stress free state. The nonlinearity is based on the assumption that the segment length is not changed by deformation. The strain tensor characterizing the deformation of segments satisfies the slip equation of the slip theory in the range of linear theory. ${ }^{32}$ The nonlinear equation satisfied by the strain tensor is a natural extension of the linear slip equation. The constitutive equation eq 16 has a simple form and is easily applied to some practical problems.

The relaxation modulus is the product of the time dependent and strain dependent terms for both shear and elongational deformation, as was found on the basis of experimental evidence. ${ }^{17-19}$

At the onset of steady shear flow, the shear stress shows stress overshoot but not the normal stress. This is at variance with experimental evidence for normal stress overshoot. ${ }^{21.45}$

At the onset of elongational steady flow, the stress increases monotonously and tends to take on a steady value, which is low at high elongation rate. In some experiments, this tendency was not found. ${ }^{43}$ The experimental results for stress growth of the elongational flow showed complex features ${ }^{39-43}$ that could not be explained by presently available theory.

\section{APPENDIX I}

We consider the linear term of eq 13

$$
\frac{\mathrm{d} \boldsymbol{a}}{\mathrm{d} t}=-\frac{1}{\tau}(\boldsymbol{a}-\mathbf{1})+\dot{\gamma} \cdot \boldsymbol{a}+\boldsymbol{a} \cdot \dot{\gamma}^{+}
$$

Equation AI.1 is satisfied by

$$
a(t)=\int_{-\infty}^{t} \frac{\mathrm{e}^{-\left(t-t^{\prime}\right) / \tau}}{\tau} \lambda\left(t, t^{\prime}\right) \mathrm{d} t^{\prime}
$$

As an approximate solution of eq 13, we find a scalar function $A\left(t, t^{\prime}\right)$ defined by

$$
\left.\begin{array}{l}
\alpha(t)=\int_{-\infty}^{t} A\left(t, t^{\prime}\right) \frac{\mathrm{e}^{-\left(t-t^{\prime}\right) / \tau}}{\tau} \lambda\left(t, t^{\prime}\right) \mathrm{d} t^{\prime} \\
A(t, t)=1
\end{array}\right\}
$$

The substitution of eq AI. 3 for $\alpha$ in eq 13 gives

$$
\begin{aligned}
& \int_{-\infty}^{t} \frac{\partial}{\partial t} A\left(t, t^{\prime}\right) \frac{\mathrm{e}^{-\left(t-t^{\prime}\right) / \tau}}{\tau} \operatorname{Tr} . \lambda\left(t, t^{\prime}\right) \mathrm{d} t^{\prime} \\
& =-\operatorname{Tr} .\left\{\left(\dot{\gamma}+\dot{\gamma}^{+}\right) \cdot \boldsymbol{\alpha}\right\}
\end{aligned}
$$

since $\operatorname{Tr} . \boldsymbol{\alpha}=3 \boldsymbol{u} \cdot \boldsymbol{u}=3$. From eq AI3, eq AI.4 leads to

$$
\begin{aligned}
& \int_{-\infty}^{t} \frac{\partial A\left(t, t^{\prime}\right)}{\partial t} \frac{\mathrm{e}^{-\left(t-t^{\prime}\right) / t}}{\tau} \operatorname{Tr} . \lambda\left(t, t^{\prime}\right) \mathrm{d} t^{\prime} \\
& =-\int_{-\infty}^{t} A\left(t, t^{\prime}\right) \frac{\mathrm{e}^{-\left(t-t^{\prime}\right) / \tau}}{\tau} \frac{\partial}{\partial t}\left\{\operatorname{Tr} . \lambda\left(t, t^{\prime}\right)\right\} \mathrm{d} t^{\prime}
\end{aligned}
$$

Equation AI.5 is satisfied by

$$
\frac{1}{A\left(t, t^{\prime}\right)} \frac{\partial A\left(t, t^{\prime}\right)}{\partial t}=-\frac{1}{\operatorname{Tr} . \lambda\left(t, t^{\prime}\right)} \frac{\partial\left(\operatorname{Tr} . \lambda\left(t, t^{\prime}\right)\right)}{\partial t}
$$

namely

$$
A\left(t, t^{\prime}\right)=3 / \operatorname{Tr} . \lambda\left(t, t^{\prime}\right)
$$

since $\operatorname{Tr} . \lambda\left(t^{\prime}, t^{\prime}\right)=3$, and eq 16 is obtained. 


\section{S. HAYASHI}

\section{APPENDIX II}

When we use the tube model for the motion of a polymer chain, we obtain the same constitutive equation as that obtained by Doi et al. ${ }^{27}$

We use the tube model with length $L$, and the motion of polymer molecules in concentrated systems is represented by a diffusive motion of the primitive chain in the tube. Let the number of segments in a polymer molecule be $n$, the end-to-end vector of the $i$ th segment be $\boldsymbol{r}_{i}$ which has the constant length $a$, and the number of polymer molecules in a unit volume be $c$. The stress $\sigma(t)$ is then obtained as

$$
\boldsymbol{\sigma}(t)=\frac{3 c k T}{a^{2}} \sum_{i=1}^{n}\left\langle\left(\boldsymbol{r}_{i} \boldsymbol{r}_{i}\right)\right\rangle-P \mathbf{1}
$$

where $\langle>$ is the average with respect to the configuration of a primitive chain and (rr) is a dyad. Since $\boldsymbol{r}_{i}$ has a constant length, the magnitude of the unit vector, $\boldsymbol{u}_{i}=\boldsymbol{r}_{i} / a$, is kept constant during deformation, and eq AII.1 can be rewritten using $\boldsymbol{u}_{i}$. Instead of the number $i$ we use the arc length $s$ of tube, $s=i a$ and obtain

$$
\boldsymbol{\sigma}(t)=\frac{3 c k T}{a} \int_{0}^{L}\langle(\boldsymbol{u}(s, t) \boldsymbol{u}(s, t))\rangle \mathrm{d} s-P \mathbf{1} \text { (AII.2) }
$$

We define the strain tensor as

$$
\boldsymbol{\alpha}(s, t)=3\langle(\boldsymbol{u}(s, t) \boldsymbol{u}(s, t))\rangle
$$

and the stress tensor is given from eq AII. 2 as

$$
\boldsymbol{\sigma}(t)=\frac{c k T}{a} \int_{0}^{L} \alpha(s, t) \mathrm{d} s-P \mathbf{1}
$$

To obtain a constitutive equation, it would be convenient to know the revolution of $\alpha(s, t)$ with time. When the deformation of the system changes with time, the tube surrouding a primitive chain is also deformed, and the motion of primitive chain is affected by both the deformation and repetition in the tube.

By the repetition, $\boldsymbol{u}(s, t)$ is transformed to $\boldsymbol{u}(s+\Delta s, t)$ after a time interval $\Delta t$ by deformation to $\boldsymbol{u}^{\prime}(s, t)$, where

$$
\boldsymbol{u}^{\prime}(s, t)=\frac{\gamma(t+\Delta t, t) \cdot \boldsymbol{u}(s, t)}{|\gamma(t+\Delta t, t) \cdot \boldsymbol{u}(s, t)|}
$$

Therefore, $\boldsymbol{u}(s, t)$ is transformed to $\boldsymbol{u}^{\prime}(s+\Delta s, t)$ after $\Delta t$; that is,

$$
\boldsymbol{u}(s, t+\Delta t)=\boldsymbol{u}^{\prime}(s+\Delta s, t)
$$

and the strain tensor satisfies the relation

$$
\boldsymbol{\alpha}(s, t+\Delta t)=\boldsymbol{\alpha}^{\prime}(s+\Delta s, t)
$$

where $\boldsymbol{\alpha}^{\prime}(s, t)$ is the strain tensor in which $\boldsymbol{u}(s, t)$ is replaced by $\boldsymbol{u}^{\prime}(s, t)$ in eq AII.3. From eq AII.5 and eq AII.7, we have

$$
\begin{aligned}
\alpha(s, t+\Delta t)= & \alpha(s, t)+\{\dot{\gamma} \cdot \alpha(s+\Delta s, t) \\
& \left.+\alpha(s+\Delta s, t) \cdot \dot{\gamma}^{+}\right\} \Delta t \\
& -\operatorname{Tr} .\left\{\left(\dot{\gamma}+\dot{\gamma}^{+}\right) \cdot \alpha(s+\Delta s, t) / 3\right\} \\
& \times \alpha(s+\Delta s, t) \Delta t
\end{aligned}
$$

Expanding eq AII. 8 with respect to $\Delta s$ and $\Delta t$ and using the relation $\left\langle\Delta s^{2}\right\rangle / 2 \Delta t=D$, we have

$$
\begin{aligned}
\frac{\partial}{\partial t} \alpha(s, t)= & D \frac{\partial^{2}}{\partial s^{2}} \alpha(s, t)+\dot{\gamma} \cdot \alpha(s, t)+\alpha(s, t) \cdot \dot{\gamma}^{+} \\
& -\operatorname{Tr} .\left\{\left(\dot{\gamma}+\dot{\gamma}^{+}\right) \cdot \alpha(s, t) / 3\right\} \cdot \alpha(s, t)
\end{aligned}
$$

where $D$ is the diffusion constant of a primitive chain and proportional to $L$. The boundary and initial conditions are

$$
\left.\begin{array}{lr}
\alpha(0, t)=\alpha(L, t)=1 & \text { boundary condition } \\
\lim _{t \rightarrow \infty} \alpha(s, t)=1 & \text { initial condition }
\end{array}\right\}
$$

When $L$ is long, eq AII.10 is a good approximation.

To integrate eq AII.9, we introduce $\alpha_{\mathrm{p}}(t)$ defined by

$$
\alpha(s, t)-1=\sum_{\mathrm{p}: \text { odd }} \frac{4}{p \pi} \sin \left(\frac{p \pi s}{L}\right)\left(\alpha_{\mathrm{p}}-1\right)
$$

(AII.11)

Using the well known 'relation 


$$
\sum_{\mathrm{p}: \text { odd }} \sin \left(\frac{p \pi s}{L}\right) / p=\frac{\pi}{4} ; \quad(0<s<L)
$$

(AII.12)

we have

$$
\left.\begin{array}{rl}
\frac{\mathrm{d}}{\mathrm{d} t} \boldsymbol{\alpha}_{\mathrm{p}}= & -\frac{1}{\tau_{\mathrm{p}}}\left(\boldsymbol{\alpha}_{\mathrm{p}}-\mathbf{1}\right)+\dot{\gamma} \cdot \boldsymbol{\alpha}_{\mathrm{p}}+\boldsymbol{\alpha}_{\mathrm{p}} \cdot \dot{\gamma}^{+} \\
& -\operatorname{Tr} .\left\{\left(\dot{\gamma}+\dot{\gamma}^{+}\right) \cdot \boldsymbol{\alpha}_{\mathrm{p}} / 3\right\} \cdot \boldsymbol{\alpha}_{\mathrm{p}} \\
\tau_{\mathrm{p}}= & L^{2} / p^{2} \pi^{2} D
\end{array}\right\}
$$

where $\tau_{p}$ is the relaxation time related to $\alpha_{p}$. Equation AII.13 is the same as eq 13. Using eq AII.11 and eq AII.12, the initial condition is rewitten as

$$
\lim _{t \rightarrow \infty} \alpha(t)=1
$$

The approximate solution of eq AII.13 becomes

$$
\alpha_{\mathrm{p}}(t)=\int_{-\infty}^{t} \frac{\mathrm{e}^{-\left(t-t^{\prime}\right) / \tau_{\mathrm{p}}}}{\tau_{\mathrm{p}}} \cdot \frac{3 \lambda\left(t, t^{\prime}\right)}{\operatorname{Tr} \cdot \lambda\left(t, t^{\prime}\right)} \mathrm{d} t^{\prime}
$$

and we have the constitutive equation

$$
\begin{aligned}
\boldsymbol{\sigma}(t)= & G_{0} \sum_{\mathrm{p}: \text { odd }} \frac{8}{p^{2} \pi^{2}} \\
& \times \int_{-\infty}^{t} \frac{\mathrm{e}^{-\left(t-t^{\prime}\right) / \tau_{\mathrm{p}}}}{\tau_{\mathrm{p}}} \cdot \frac{3 \lambda\left(t, t^{\prime}\right)}{\operatorname{Tr} \cdot \lambda\left(t, t^{\prime}\right)} \mathrm{dt}^{\prime}-P \mathbf{1} \\
\mathrm{G}_{0}= & c k T L / a
\end{aligned}
$$

(AII.16)

This is an extension of the formula of eq 16 to a system consisting of tube model, and is a somewhat simplified expression of that obtained by Doi et al. ${ }^{27}$

\section{REFERENCES}

1. A. S. Lodge, Rheol. Acta, 7, 379 (1968).

2. A. S. Lodge, "Elastic Liquids," Academic Press, New York, 1964.

3. J. L. White and A. B. Metzner, J. Polym. Sci., 7, 1867 (1963).

4. R. B. Bird, Chem. Eng. Prog. Symp. Sec., 58, 61 (1965).

5. T. W. Spriggs, Chem. Eng. Sci., 20, 931 (1965).
6. R. B. Bird and P. J. Carreau, Chem. Eng. Sci, 23, 437 (1968).

7. L. J. Zapas and J. C. Philps, J. Res. Nat. Bur. Stand., 75A, 93 (1971).

8. M. Yamamoto, J. Phys. Soc. Jpn., 25, 239 (1968).

9. M. Yamamoto, Appl. Polym. Symp., 23, 3 (1973).

10. M. Yamamoto, J. Soc. Mater. Sci. Jpn., 21, 355 (1972).

11. M. Yamamoto, Trans. Soc. Rheol., 15, 331 (1971).

12. M. Takahashi and S. Onogi, J. Soc. Rheol. Jpn., 2, 95 (1974); ibid., 3, 1 (1975).

13. T. W. Spriggs, J. D. Huppler, and R. B. Bird, Trans. Soc. Rheol., 10, 191 (1965).

14. M. Takahashi, T. Masuda, and S. Onogi, J. Soc. Rheol. Jpn., 1, 16 (1973).

15. K. Osaki, M. Fukuda, S. Ota, and M. Kurata, J. Soc. Rheol. Jpn., 2, 106 (1974).

16. M. Fukuda, K. Osaki, and M. Kurata, J. Soc. Rheol. Jpn., 2, 110 (1974).

17. Y. Einaga, K. Osaki, M. Kurata, S. Kimura, and M. Tamura, Polym. J., 2, 550 (1971).

18. Y. Einaga, K. Osaki, M. Kurata, S. Kimura, N. Yamada, and M. Tamura, Polym. J., 5, 91 (1973).

19. K. Osaki, Y. Einaga, M. Kurata, N. Yamada, and M. Tamura, Polym. J., 5, 283 (1973).

20. M. Yamamoto, "Mechanics of Body Deformation" (in Japanese), Seibundo Shinkosha Publishing Co., Tokyo, 1972.

21. R. B. Bird, R. C. Armstrong, and O. Hassager, "Dynamics of Polymer Liquids," Vol. I, II, Wiley, New York, 1976.

22. G. Astarita and G. Marricci, "Principle of NonNewtonian Fluid Mechanics," McGraw-Hill, London, 1972.

23. A. S. Lodge, "Body Tensor Fields in Continum Mechanics," Academic Press, New York, 1974.

24. W. W. Grassley, Advan. Polym. Sci., 16, 1 (1974).

25. M. Doi, Kobunshi, 27, 649 (1978).

26. M. Doi, Physique, 36, 607 (1975).

27. M. Doi and S. F. Edwards, J. Chem. Soc., Faraday Trans. 2, 74, 560, 918, 1802 (1978); ibid., 75, 38 (1979).

28. M. Yamamoto, J. Phys. Soc. Jpn., 11, 413 (1956); ibid., 12, 1148 (1957); ibid., 13, 1200 (1958).

29. A. S. Lodge, Trans. Faraday Soc., 52, 120 (1959).

30. K. W. Scott and R. S. Stein, J. Chem. Phys., 21, 1281 (1959).

31. S. Hayashi, Prog. Theor. Phys., Suppl., 10, 82 (1959).

32. S. Hayashi, J. Phys. Soc. Jpn., 18, 131 (1963); ibid., 19, 101 (1964).

33. S. Hayashi and Y. Takano, J. Phys. Soc. Jpn., 23, 1063 (1967).

34. Y. Takano, Polym. J., 6, 61 (1974).

35. Y. Takano, J. Soc. Rheol. Jpn., 2, 87 (1974).

36. S. Hayashi, J. Soc. Rheol. Jpn., 3, 159 (1975).

37. S. Hayashi, Polym. J., 10, 59 (1978); ibid., 13, 693 (1981). 


\section{S. HAYASHI}

38. S. Hayashi, J. Macromol. Sci. Phys., B19, 632 (1981).

39. N. E. Hudson and J. Ferguson, Trans. Soc. Rheol., 20, 265 (1976).

40. H. Munstedt, Trans. Soc. Rheol., 23, 421 (1979).

41. R. N. Shroff, L. V. Vacio, and M. Shida, Trans. Soc. Rheol., 21, 429 (1977).

42. R. S. Stratton, J. Colloid. Sci., 22, 517 (1966).
43. J. Meissner, Rheol. Acta, 10, 230 (1971).

44. S. Hayashi, Repts. Prog. Polym. Phys. Jpn., 21, 97 (1978); 23, 113 (1980).

45. S. Hayashi, Rep. Prog. Polym. Phys. Jpn., 26, 95, 99 (1983).

46. J. D. Huppler, I. F. Macdonald, E. Ashare, T. W. Spriggs, R. B. Bird, and L. A. Holmes, Trans. Soc. Rheol., 11, 181 (1967). 